(1)

CrossMark

\title{
Celebrating World Tuberculosis Day at the time of COVID-19
}

\author{
To the Editor:
}

World Tuberculosis Day, observed on 24 March each year, is the global call to raise awareness, celebrate successes and remind the world that tuberculosis (TB) is still the number one killer among the infectious causes of death [1]. TB is a preventable and curable disease, and its control is a highly cost-effective health intervention. However, diagnostic delay and inadequate treatment contribute to the severity and mortality of the disease as well as the risk of transmission and development of drug resistance. Despite the fact that TB disproportionately impacts low- and middle-income countries, it does not spare any country in the world, including those in the European Union/European Economic Area, where more than 4000 people still die from the disease every year [2]. Moreover, the high number of multidrug-resistant (MDR)-TB cases in some countries of the World Health Organization (WHO) European Region poses a growing public health threat. Despite the sheer numbers, TB has for too long been overshadowed by HIV and malaria, and currently by the novel coronavirus severe acute respiratory syndrome coronavirus 2 (SARS-CoV-2) responsible for the coronavirus disease 2019 (COVID-19) epidemic.

In Italy, and in particular in Lombardy, the number of COVID-19 cases rose to 17713 on Tuesday 18 March, 2020, from 984 announced on 1 March, forcing health authorities to put in place "draconian" measures to contain the spread of infection. It is unclear if these measures will be enforced and will succeed, but for the time being the priority is to stop virus transmission and avoid the collapse of the national health system, which is currently overwhelmed in the face of this urgent demand. This year, all TB day events scheduled in Italy will be cancelled due to the spread of COVID-19. Nevertheless, it is necessary to keep raising awareness of the global TB problem. The COVID-19 epidemic offers the opportunity to make some assessments on the shared aspects of COVID-19 and TB, as well as the challenges and lessons learned from the control efforts of each of them that could be of mutual benefit (figure 1).

The major common thread linking COVID-19 and TB (figure 1) is that both, in different ways, have natural capacities to stress healthcare systems. The recent epidemic of COVID-19 in Italy highlights how a rapid spread of a serious epidemic can severely affect healthcare systems anywhere in the world. Shortages of personal protective devices, mechanical ventilators, and worse, beds in intensive care units are a serious sign that a health system cannot adapt rapidly to a health emergency [3]. On the contrary, TB is a "silent" epidemic [4]. The New York epidemic in the early 1990s taught us that as soon as TB drops off the radar of public health and politics, the effects can be catastrophic on its epidemiology and the resulting public spending. By 1990, New York City accounted for more than $15 \%$ of the country's cases and the federal government needed to spend more than USD 1 billion to contain the MDR-TB outbreak [5]. Likewise, COVID-19 will lead to devastating economic effects even for the wealthiest regions of Italy that are currently affected.

A second similarity is the importance of timely and rapid diagnosis and public awareness for infectious disease control. Long-standing struggles in TB diagnosis represent one of the main reasons for on-going disease transmission in many settings [6,7]. Recent clusters of MDR-TB in Italy and Europe have been linked to diagnostic delays together with inappropriate treatment [8], mainly attributed to the lack of expertise and clinical suspicion on a disease perceived as disappeared. It is not unlikely, despite its emergence in China in December 2019, that many countries around the world underestimated the seriousness of the rapidly expanding COVID-19 epidemic [9]. Italy itself experienced a delay in recognising the severity of the outbreak and in taking the appropriate measures.

Essential components of infectious disease control are adequate training of health workers in recognising a disease and an effective surveillance system in place to monitor its behaviour [10]. This requires time of COVID-19. Eur Respir J 2020; 55: 2000650 [https://doi.org/10.1183/13993003.00650-2020]. 
coordination from the local clinic level to the regional and central authorities, backed by adequate financial and human resources.

A third similarity is that both diseases carry a perceivable social stigma. On one side, TB is a "model" of stigma because of its associations with immigration from other countries, low social class, HIV, drug use, alcohol abuse and poverty.

On the other side, with the recent spread of COVID-19 in Italy, one can now confirm that holding an Italian passport may mean denial of access to a large number of countries and a clearly perceived stigma.

A fourth similarity is the limited knowledge of individual's susceptibility to infection. Clearly, to improve prevention and control of infectious diseases, it is critical to understand why some individuals are susceptible to infectious diseases while others, acquiring the same pathogen, remain asymptomatic. In TB, genetic and non-genetic factors of both host and pathogen play an important role in the development of the disease [11]. Yet, little is known about these factors that, ultimately, may provide some clues for the future development of vaccines, therapies and novel drug targets. In the case of COVID-19, despite the recently found virus entry route, it is not clear whether the differences in viral receptor-binding domain structure contribute to differences in infectivity, transmissibility and disease severity [12].

Lastly, there is a major lack of data-sharing platforms that accelerate collaborative research discovery. As for TB, COVID-19, is still characterised by a patchwork of fragmented data with lack of coordination at national and international level which creates barriers to gathering informative datasets useful to shape preventive and control measures, as well as facilitate research.

Not surprisingly, there are also differences between the two epidemics. For TB, the long-standing scarcity of investments in the surveillance system and the lack of a uniform policy on TB control enforced in all regions of Italy has led to weak reporting of national TB data to the European Centre for Disease Control (ECDC) and WHO, with a resulting insufficient understanding of the burden of TB in the country. In contrast, a rapid and unprecedented mobilisation of resources to contain the COVID-19 epidemic is leading to a coordinated national surveillance system able to monitor epidemic intensity, the impact on the population and healthcare system and assess measures in place.

Finally, COVID-19 and TB have an additional major difference. COVID-19's future may move into two distinct directions: either SARS-CoV-2 is eradicated, as was the case of SARS in 2003, or humanity will have to coexist with it until a vaccine is becomes available. At the same time, TB remains a long-standing public health problem producing more than 10 new cases and more than one death every day in Italy [13]. Its elimination cannot be achieved until countries commit to implement effective surveillance systems and other core measures [14]. The lack of consistent policies is a major Achilles' heel rendering Italy
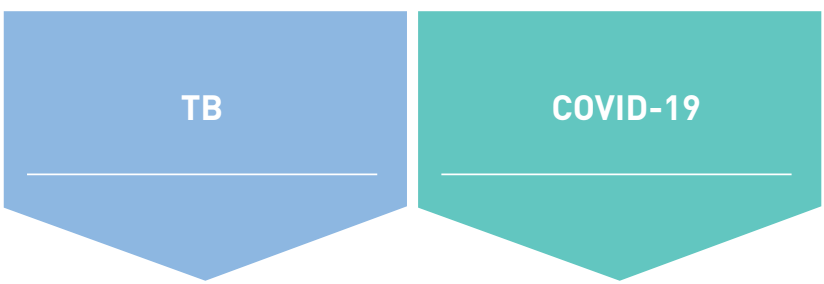

Overload of national health system

Need rapid diagnosis and public awareness

Lead to social stigma

Lack of knowledge on individual susceptibility

Lack of data sharing platform

Lack of investments for

national surveillance

systems

Lack of consistent

national policies
Appropriate health

surveillance

systems used to monitor

and track the epidemic

Fast adoption of national policies to contain epidemic and differences betwee disease 2019 (COVID-19) and tuberculosis (TB) 
(and other countries) vulnerable to those TB outbreaks that regularly occur (often neglected by the media and the population) in schools, hospitals and other care facilities. In turn, these outbreaks will keep favouring $M$. tuberculosis transmission in the community rendering unattainable the achievements of the UN Sustainable Development Goals (SDG).

In conclusion, virtually all infectious diseases are preventable. However, some of them, such as COVID-19, will be hopefully contained quickly thank to a global rapid response backed by huge political and financial commitments. For some others, we will still need to commemorate through a dedicated "world day" to be able to raise public awareness and mobilise proper resources.

Riccardo Alagna ${ }^{1}$, Giorgio Besozzi ${ }^{2,3}$, Luigi Ruffo Codecasa ${ }^{3}$, Andrea Gori ${ }^{4,5}$, Giovanni Battista Migliori $\odot^{6}$, Mario Raviglione ${ }^{7}$ and Daniela Maria Cirillo ${ }^{1}$

${ }^{1}$ IRCCS San Raffaele Scientific Institute, Milan, Italy. ${ }^{2}$ StopTB Italia Onlus, Milan, Italy. ${ }^{3}$ Regional TB Reference Centre, Istituto Villa Marelli, Ospedale Niguarda, Milan, Italy. ${ }^{4}$ Infectious Diseases Unit, IRCCS Ca' Granda Ospedale Maggiore Policlinico Foundation, Milan, Italy. ${ }^{5}$ Dept of Pathophysiology and Transplantation, University of Milano, Milan, Italy.

${ }^{6}$ Servizio di Epidemiologia Clinica delle Malattie Respiratorie, Istituti Clinici Scientifici Maugeri IRCCS, Tradate, Italy.

${ }^{7}$ Global Health Centre, University of Milan, Milan, Italy.

Correspondence: Daniela Maria Cirillo, IRCCS San Raffaele Scientific Institute, Via Olgettina 60, Milan 20132, Italy. E-mail: cirillo.daniela@hsr.it

Received: 11 March 2020 | Accepted after revision: 24 March 2020

Conflict of interest: None declared.

\section{References}

1 Global Tuberculosis Report, 2019. Geneva, World Health Organization, 2019. Available from https://apps.who.int/ iris/bitstream/handle/10665/329368/9789241565714-eng.pdf?ua=1

2 Merk H, Kodmon C, van der Werf MJ. Will we reach the Sustainable Development Goals target for tuberculosis in the European Union/European Economic Area by 2030? Euro Surveill 2019; 24.

3 Wang X, Zhang X, He J. Challenges to the system of reserve medical supplies for public health emergencies: reflections on the outbreak of the severe acute respiratory syndrome coronavirus 2 (SARS-CoV-2) epidemic in China. Biosci Trends 2020; 14: 3-8.

4 Graber-Stiehl I. The silent epidemic killing more people than HIV, malaria or TB. Nature 2018; 564: 24-26.

5 Landesman SH. Commentary: tuberculosis in New York City-the consequences and lessons of failure. Am J Public Health 1993; 83: 766-768.

6 Temesgen Z, Cirillo DM, Raviglione MC. Precision medicine and public health interventions: tuberculosis as a model? Lancet Public Health 2019; 4: e374.

7 Migliori GB, Nardell E, Yedilbayev A, et al. Reducing tuberculosis transmission: a consensus document from the World Health Organization Regional Office for Europe. Eur Respir J 2019; 53: 1900391.

8 Walker TM, Merker M, Knoblauch AM, et al. A cluster of multidrug-resistant Mycobacterium tuberculosis among patients arriving in Europe from the Horn of Africa: a molecular epidemiological study. Lancet Infect Dis 2018; 18: 431-440.

9 The Lancet. COVID-19: too little, too late? Lancet 2020; 395: 755.

10 Storr J, Twyman A, Zingg W, et al. Core components for effective infection prevention and control programmes: new WHO evidence-based recommendations. Antimicrob Resist Infect Control 2017; 6: 6.

11 Parvati Sai Arun PV, Miryala SK, Rana A, et al. System-wide coordinates of higher order functions in host-pathogen environment upon Mycobacterium tuberculosis infection. Sci Rep 2018; 8: 5079.

12 Zhou P, Yang XL, Wang XG, et al. A pneumonia outbreak associated with a new coronavirus of probable bat origin. Nature 2020; 579: 270-273.

13 WHO Regional Office for Europe/European Centre for Disease Prevention and Control. Tuberculosis Surveillance and Monitoring in Europe 2019 - 2017 Data. Copenhagen, WHO Regional Office for Europe, 2019. Available from www.ecdc.europa.eu/en/publications-data/tuberculosis-surveillance-and-monitoring-europe-2019

14 Lonnroth K, Migliori GB, Abubakar I, et al. Towards tuberculosis elimination: an action framework for low-incidence countries. Eur Respir J 2015; 45: 928-952. 